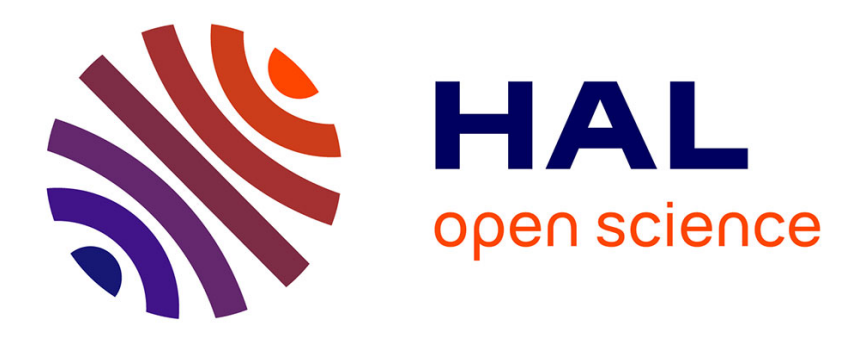

\title{
An Integral Representation Theorem of g-expectations
}

Chen Zengjing, Agnès Sulem

\section{To cite this version:}

Chen Zengjing, Agnès Sulem. An Integral Representation Theorem of g-expectations. [Research Report] RR-4284, INRIA. 2001. inria-00072303

\section{HAL Id: inria-00072303 https://hal.inria.fr/inria-00072303}

Submitted on 23 May 2006

HAL is a multi-disciplinary open access archive for the deposit and dissemination of scientific research documents, whether they are published or not. The documents may come from teaching and research institutions in France or abroad, or from public or private research centers.
L'archive ouverte pluridisciplinaire HAL, est destinée au dépôt et à la diffusion de documents scientifiques de niveau recherche, publiés ou non, émanant des établissements d'enseignement et de recherche français ou étrangers, des laboratoires publics ou privés. 


\section{An integral representation theorem of g-expectations}

Zengjing Chen and Agnès Sulem

\section{$\mathbf{N}^{\circ} \mathbf{4 2 8 4}$}

October, 182001

THÈME 4 



\title{
An integral representation theorem of $g$-expectations
}

\author{
Zengjing Chen* and Agnès Sulem ${ }^{\dagger}$ \\ Thème 4 - Simulation et optimisation \\ de systèmes complexes \\ Projet MATHFI
}

Rapport de recherche $\mathrm{n}^{\circ} 4284$ - October, 182001 - 20 pages

\begin{abstract}
The notion of $g$-expectations was first introduced by S.Peng via a class of nonlinear backward stochastic differential equations (BSDEs). He showed that $g$-expectations preserve many of the basic properties of the convenient mathematical expectations and conditional expectations except linearity. He also raised the question about the integral representation of $g$-expectations. In this paper, we try to investigate Peng's question and give a necessary and sufficient condition under which $g$-expectations can be represented by a Choquet integral.
\end{abstract}

Key-words: BSDE, $g$-expectation, convex capacity, Choquet integral, integral representation theorem.

* Department of Mathematics, Shandong University, Jinan, 250100, P.R.China, email: zchen@math.hkbu.edu.hk.

$\dagger$ INRIA-Rocquencourt, email: agnes.sulem@inria.fr 


\section{Theorème de représentation intégrale pour les $g$-espérances}

Résumé : La notion de $g$-espérance a été introduite par S.Peng au moyen d'une classe d'équations différentielles stochastiques rétrogrades. La $g$-espérance conserve certaines propriétés de l'éspérance et de l'éspérance conditionnelle mais pas la linéarité. Dans ce papier on apporte des éléments de réponse à la question de Peng concernant la représentation intégrale de la $g$-espérance et on donne une condition suffisante sous laquelle la $g$-espérance peut être représentée par une intégrale de Choquet.

Mots-clés : EDSR, $g$-espérance, capacité, intégrale de Choquet, représentation intégrale, théorème de représentation. 


\section{Introduction}

The first published paper on nonlinear BSDEs by Pardoux and Peng [PP1] appeared in 1990. Since then, many researchers have been working on this subject, due to the connection of this subject with mathematical finance, stochastic control, partial differential equations, stochastic games, stochastic geometry and mathematical economics (see for example [KPQ, KQ, P1, P2, P3, H, MPY, HL, D, CE] and references therein). More recently, Peng [P1, P2 ] introduced the notions of $g$-expectations, conditional $g$-expectations and the corresponding $g$-martingales via BSDEs and set up a connection between BSDEs and mathematical expectations and martingales. In [P1,P2], he showed that $g$-expectations preserve many of the basic properties of the mathematical expectations and martingales except linearity (see $[\mathrm{P} 1, \mathrm{P} 2, \mathrm{CP}]$ for details).

It is well-known that for a given probability measure $P$, the expectation $E \xi$ of a random variable $\xi$, can be computed as

$$
E \xi=\int_{-\infty}^{0}[P(\xi \geq t)-1] d t+\int_{0}^{\infty} P(\xi \geq t) d t
$$

The Choquet integral was first introduced in the mathematical literature by Choquet [Ch 1955]. He extended the above Riemann integral to the case where $P$ is a non-additive set function (capacity). Although the Choquet integral was originally motivated by potential theory in physics, it has found many applications in various fields. In particular, owing to Schmeidler's work [S1,S2], the Choquet integral has become an important tool in describing individuals' behavior under uncertainty in economics. A natural question is: Can $g$-expectation be represented by a Choquet integral? More precisely, let $\mathcal{E}_{g}[\xi]$ be the $g$-expectation of a random variable $\xi$ and $P_{g}(A)$ be the $g$-probability defined by $P_{g}(A):=\mathcal{E}_{g}\left[1_{A}\right]$ for a given event $A$. Under which conditions does the following integral representation hold?

$$
\mathcal{E}_{g}[\xi]=\int_{-\infty}^{0}\left[P_{g}(\xi>t)-1\right] d t+\int_{0}^{\infty} P_{g}(\xi>t) d t
$$

In this paper, we discuss this issue and provide a necessary and sufficient condition under which the integral representation $\left(^{*}\right)$ holds . The paper is organized as follows: In Section 1 , we recall briefly some basic lemmas of BSDEs and the notions of $g$-expectations and related basic properties. In Section 3, we give a necessary condition under which $g$ expectation can be represented as a Choquet integral. Fianally in Section 4, we prove that such a necessary condition is also sufficient.

$\mathrm{RR} \mathrm{n}^{\circ} 4284$ 


\section{BSDEs and $g$-expectation}

Pardoux and Peng (1990) showed an existence and uniqueness theorem for nonlinear BSDEs. Furthermore, S.Peng (1997) [P1] introduced the notions of $g$-expectations via this class of BSDEs. In this section, we shall recall the basic lemmas of BSDEs, the notion of $g$ expectation and related basic properties. To be more specific, fix $T \in[0, \infty)$, let $\left(W_{t}\right)_{0 \leq t \leq T}$ be a 1-dimensional standard Brownian motion defined on a completed probability space $(\Omega, \mathcal{F}, P)$ and $\left\{\mathcal{F}_{t}\right\}_{0 \leq t \leq T}$ be the natural filtration generated by $\left(W_{t}\right)_{0 \leq t \leq T}$, i.e.

$$
\mathcal{F}_{t}=\sigma\left\{W_{s} ; s \leq t\right\}
$$

We use the following notation:

$$
\begin{aligned}
& L^{2}(0, T):=\left\{\left(\mathcal{F}_{t}\right) \text {-adapted processes } V \text { with }\|V\|_{L^{2}}:=\left[E \int_{0}^{T}|V(s)|^{2} d s\right]^{\frac{1}{2}}<\infty\right\} \\
& L^{2}\left(\Omega, \mathcal{F}_{t}, P\right):=\left\{\left(\mathcal{F}_{t}\right) \text {-measurable random variables } \xi \text { with } E|\xi|^{2}<\infty\right\}, t \in[0, T]
\end{aligned}
$$

Let $g$ be a function from $\mathbb{R}^{2} \times[0, T]$ into $\mathbb{R}$. We shall say that $g$ satisfies hypothesis (H1) and (H2) if:

(H1) For any $(y, z) \in \mathbb{R}^{2}, g(y, z, t)$ is continuous in $t$ and

$$
\int_{0}^{T}\left|g^{2}(y, z, s)\right| d s<\infty
$$

(H2) $g$ satisfies the Lipschitz condition: there exists a constant $\mu>0$ such that

$$
\left|g\left(y_{1}, z_{1}, t\right)-g\left(y_{2}, z_{2}, t\right)\right| \leq \mu\left[\left|y_{1}-y_{2}\right|+\left|z_{1}-z_{2}\right|\right], \forall\left(y_{i}, z_{i}\right) \in \mathbb{R}^{2}, i=1,2 \text {. }
$$

Pardoux and Peng (1990) obtained the following lemma:

Lemma 1 Suppose that $g$ satisfies (H1) and (H2). For any $\xi \in L^{2}\left(\Omega, \mathcal{F}_{T}, P\right)$, there exists a unique pair of adopted process $(y, z) \in L^{2}(0, T) \times L^{2}(0, T)$, which depends on $(\xi, g, T)$, satisfying the following BSDE:

$$
y_{t}=\xi+\int_{t}^{T} g\left(y_{s}, z_{s}, s\right) d s-\int_{t}^{T} z_{s} d W_{s}, 0 \leq t \leq T
$$

The following lemma can be found in [ KPQ,P1,P2]:

Lemma 2 Suppose that $g_{1}$ and $g_{2}$ satisfy (H1) and (H2). For any $\xi_{1}, \xi_{2} \in L^{2}\left(\Omega, \mathcal{F}_{T}, P\right)$, let $\left(y^{i}, z^{i}\right)(i=1,2)$ be the solutions of BSDE (1) corresponding to $\xi=\xi_{1}$ and $g=g_{1}$, $\xi=\xi_{2}$ and $g=g_{2}$, respectively. Then there exists a constant $c>0$ such that

$$
E\left[\sup _{t \leq s \leq T}\left|y_{s}^{1}-y_{s}^{2}\right|^{2}+\int_{t}^{T}\left|z_{s}^{1}-z_{s}^{2}\right|^{2} d s \mid \mathcal{F}_{t}\right] \leq c E\left[\left|\xi_{1}-\xi_{2}\right|^{2}+\left(\int_{t}^{T}\left|\bar{g}_{s}\right| d s\right)^{2} \mid \mathcal{F}_{t}\right]
$$

where $\bar{g}_{s}:=g_{1}\left(y_{s}^{1}, z_{s}^{1}, s\right)-g_{2}\left(y_{s}^{1}, z_{s}^{1}, s\right) \mid$. 
Remark 1 Lemma 2 is one of the important lemmas of this paper. It implies that, if $\xi_{2}$ converges to $\xi_{1}$ in $L^{2}\left(\Omega, \mathcal{F}_{T}, P\right)$ and $g_{1}\left(y^{1}, z^{1}, \cdot\right)$ converges to $g_{2}\left(y^{1}, z^{1}, \cdot\right)$ in $L^{2}(0, T)$, then $\left(y^{2}, z^{2}\right)$ converges to $\left(y^{1}, z^{1}\right)$ in $L^{2}(0, T) \times L^{2}(0, T)$.

Assumption A. Let $b(t, x):[0, T] \times \mathbb{R} \rightarrow \mathbb{R}, \sigma(t, x):[0, T] \times R \rightarrow R$ be continuous in $(t, x)$ and uniformly Lipschitz continuous in $x$.

By the existence theorem of SDE, there exists a unique strong solution $\left\{X_{s}^{t, x}\right\}$ satisfying the SDE:

$$
\left\{\begin{array}{l}
d X_{s}=b\left(s, X_{s}\right) d s+\sigma\left(s, X_{s}\right) d W_{s}, \\
X_{t}=x, s \in[t, T] .
\end{array}\right.
$$

Let $\Phi(x)$ be a continuous function defined on $\mathbb{R}$ such that $\Phi\left(X_{T}^{t, x}\right) \in L^{2}\left(\Omega, \mathcal{F}_{T}, P\right)$ and $\left(y^{t, x}, z^{t, x}\right)$ be the solution of the BSDE:

$$
y_{s}=\Phi\left(X_{T}^{t, x}\right)+\int_{s}^{T} g\left(y_{r}, z_{r}, r\right) d r-\int_{s}^{T} z_{r} d W_{r}, \quad s \in[0, T] .
$$

Pardoux and Peng (1992) proved the following lemma:

Lemma 3 Suppose that the functions $b, \sigma, \Phi, g$ are $C^{3}$ with bounded derivatives, and let $\left(y_{s}^{t, x}, z_{s}^{t, x}\right)$ be the solution of BSDE (3), then

(i) $u(t, x):=y_{t}^{t, x} \in C^{1,2}([0, T] \times \mathbb{R}, \mathbb{R})$ is the unique solution of the following partial differential equation (PDE):

$$
\left\{\begin{array}{l}
\partial_{t} u(t, x)+\mathcal{L} u(t, x)+g\left(t, u(t, x), \sigma^{*}(t, x) \partial_{x} u(t, x)\right)=0 \\
u(T, x)=\Phi(x)
\end{array}\right.
$$

where $\mathcal{L} u(t, x):=\frac{1}{2} \sigma^{2}(t, x) \partial_{x}^{2} u(t, x)+b(t, x) \partial_{x} u(t, x)$,

(ii) $z_{s}^{t, x}=\sigma\left(s, X_{s}^{t, x}\right) \partial u_{x}\left(s, X_{s}^{t, x}\right)$, a.e. $s \in[t, T]$, where $\partial_{x} u$ is the partial derivative of $u$.

Using BSDE (1), Peng (1997) introduced the notion of $g$-expectation:

Definition 1 Suppose that $g$ satisfies (H1) and (H2). For any $\xi \in L^{2}\left(\Omega, \mathcal{F}_{T}, P\right)$, let $\left(y_{t}^{\xi, g, T}, z_{t}^{\xi, g, T}\right)$ be the solution of BSDE (1).

(1) We call $\mathcal{E}_{g}^{T}[\xi]$ and $\mathcal{E}_{g}^{T}\left[\xi \mid \mathcal{F}_{t}\right]$ defined by

$$
\mathcal{E}_{g}^{T}[\xi]:=y_{0}^{\xi, g, T} ; \quad \mathcal{E}_{g}^{T}\left[\xi \mid \mathcal{F}_{t}\right]:=y_{t}^{\xi, g, T}, \quad 0 \leq t \leq T,
$$

respectively the $g$-expectation and the conditional $g$-expectation of the random variable $\xi$ generated by $g$ on the time interval $[0, T]$.

$\mathrm{RR} \mathrm{n}^{\circ} 4284$ 
(2) We call $V_{t}(\xi, g)$ defined by

$$
V_{t}(\xi, g):=z_{t}^{\xi, g, T}, 0 \leq t \leq T,
$$

the volatility of the random variable $\xi$.

(3) For any $A \in \mathcal{F}_{T}$, we call $P_{g}(A)$ defined by

$$
P_{g}(A):=\mathcal{E}_{g}^{T}\left[1_{A}\right]
$$

the g-probability of the event $A$.

To simplify notation, we rewrite $\mathcal{E}_{g}^{T}[\xi], \mathcal{E}_{g}^{T}\left[\xi \mid \mathcal{F}_{t}\right]$ and $P_{g}(\cdot)$ as $\mathcal{E}_{a}^{T}[\xi], \mathcal{E}_{a}^{T}\left[\xi \mid \mathcal{F}_{t}\right]$ and $P_{a}(\cdot)$ respectively if $g$ is of the form: $g(y, z, t):=a(t)|z|$.

Remark 2 (1) Obviously, if $g=0$, then $\mathcal{E}_{g}^{T}\left[\xi \mid \mathcal{F}_{t}\right]=E\left[\xi \mid \mathcal{F}_{t}\right], \mathcal{E}_{g}^{T}[\xi]=E \xi$ and $P_{g}(A)=$ $P(A)$.

(2) The reason why $V_{t}(\xi, g)$ is called volatility is because BSDE (1) can be rewritten as

$$
\mathcal{E}_{g}^{T}\left[\xi \mid \mathcal{F}_{t}\right]=\mathcal{E}_{g}^{T}[\xi]-\int_{0}^{t} g\left(y_{s}, z_{s}, s\right) d s+\int_{0}^{t} V_{s}(\xi, g) d W_{s},
$$

which is the Martingale Representation Theorem if $g \equiv 0$. This notation will be very convenient to prove the theorems in next section.

(3) The $P_{g}(\cdot)$-probability is a nonlinear probability measure.

By Definition $1,\left(\mathcal{E}_{g}^{T}\left[\xi \mid \mathcal{F}_{t}\right], V_{t}(\xi, g)\right)$ consists of a pair solution of BSDE (1). For convenience, in this paper, we shall use $(y, z)$ and $\left(\mathcal{E}_{g}^{T}\left[\xi \mid \mathcal{F}_{t}\right], V_{t}(\xi, g)\right)$ alternately to express the solution of BSDE (1).

Remark 3 (1) The g-expectation $\mathcal{E}_{g}^{T}[\cdot]$, considered as a nonlinear operator on $L^{2}\left(\Omega, \mathcal{F}_{T}, P\right)$, usually depends not only on $g$ but also on $T$. That is, if $\xi$ is $\mathcal{F}_{r}$-measurable, it is certainly $\mathcal{F}_{t}$-measurable for all $t>r$, but the equality

$$
\mathcal{E}_{g}^{T}[\xi]=\mathcal{E}_{g}^{t}[\xi], \quad 0 \leq r \leq t \leq T,
$$

may fail.

For example, let $g=1$, then $\mathcal{E}_{g}^{T}[\xi]=E \xi+T$, and $\mathcal{E}_{g}^{r}[\xi]=E \xi+r$, thus if $T \neq r$, then $\mathcal{E}_{g}^{T}[\xi] \neq \mathcal{E}_{g}^{r}[\xi]$. That is, $\mathcal{E}_{g}^{T}[\cdot]$ depends on the time interval $[0, T]$. 
(2) The mathematical expectation $E[\cdot]$, viewed as a linear operator on $L^{1}\left(\Omega, \mathcal{F}_{T}, P\right)$, has the property that $E\left[a \mid \mathcal{F}_{t}\right]=$ a for all constant $a \in \mathbb{R}$. The property usually is not true for $\mathcal{E}_{g}[\cdot]$. The following lemma provides conditions under which the g-expectation preserves such a property,

Lemma 4 The following conditions are equivalent:

(1) $g(y, 0, t)=0$, for all $(y, t) \in \mathbb{R} \times[0, T]$;

(2) $\mathcal{E}_{g}^{T}\left[a \mid \mathcal{F}_{t}\right]=a$ for all $a \in \mathbb{R}$

(3) $\eta:=\mathcal{E}_{g}^{T}\left[\xi \mid \mathcal{F}_{t}\right]$ is the unique $\mathcal{F}_{t}$-measurable random variable such that

$$
\mathcal{E}_{g}^{T}\left[1_{A} \xi\right]=\mathcal{E}_{g}^{T}\left[1_{A} \eta\right], \quad \forall A \in \mathcal{F}_{t}, \quad t \in[0, T]
$$

Proof. $(1) \Rightarrow(3)$ follows from $[\mathrm{P} 1] ;(3) \Rightarrow(2)$ is obvious.

Now let us prove $(2) \Rightarrow(1)$ :

For any $a \in R$, let $y_{t}:=\mathcal{E}_{g}^{T}\left[a \mid \mathcal{F}_{t}\right]$, then by the definition of $\mathcal{E}_{g}^{T}[\cdot],\left(y_{t}\right)$ is the solution of the BSDE:

$$
y_{t}=a+\int_{t}^{T} g\left(y_{s}, z_{s}, s\right) d s-\int_{t}^{T} z_{s} d W_{s}, 0 \leq t \leq T .
$$

It follows by $y_{t}=a$ that we have

$$
\int_{t}^{T} g\left(a, z_{s}, s\right) d s-\int_{t}^{T} z_{s} d W_{s}=0, \quad \forall t \in[0, T] .
$$

Consequently, $z_{t}=0$, a.e. $t \in[0, T]$ and $g(a, 0, t)=0$, a.e.t $\in[0, T]$.

$>$ From now on, we assume that $g$ satisfies

$$
g(y, 0, t)=0, \forall(y, t) \in \mathbb{R} \times[0, T] .
$$

Lemma 5 For any $r \in[0, T]$, if $\xi \in L^{2}\left(\Omega, \mathcal{F}_{r}, P\right)$ and $g$ satisfies (H3), then

$$
\mathcal{E}_{g}^{T}[\xi]=\mathcal{E}_{g}^{\tau}[\xi], \quad \tau \in[r, T] .
$$

Proof. let $\left(\mathcal{E}_{g}^{\tau}\left[\xi \mid \mathcal{F}_{t}\right], z_{s}^{\tau}\right)$ be the solution of the BSDE:

$$
y_{t}^{\tau}=\xi+\int_{t}^{\tau} g\left(y_{s}^{\tau}, z^{\tau}, s\right) d s-\int_{t}^{\tau} z_{s}^{\tau} d W_{s}
$$

$\mathrm{RR} \mathrm{n}^{\circ} 4284$ 
It is easy to check that if $g$ satisfies (H3), then $\left(y_{t}, z_{t}\right)$ defined by

$$
y_{t}:=\left\{\begin{array}{ll}
\xi, & t \geq \tau ; \\
\mathcal{E}_{g}^{\tau}\left[\xi \mid \mathcal{F}_{t}\right], & t<\tau .
\end{array} \quad z_{t}:= \begin{cases}0, & t \geq \tau ; \\
z_{t}^{\tau}, & t<\tau .\end{cases}\right.
$$

is the solution of BSDE:

$$
y_{t}=\xi+\int_{t}^{T} g\left(y_{s}, z, s\right) d s-\int_{t} z_{s} d W_{s} .
$$

By the uniqueness of the solution of the BSDE,

$$
\mathcal{E}_{g}^{T}\left[\xi \mid \mathcal{F}_{t}\right]=\mathcal{E}_{g}^{\tau}\left[\xi \mid \mathcal{F}_{t}\right], \quad t \leq r \leq \tau \leq T .
$$

In particular, if $t=0$, we complete the proof.

Under assumption (H3), applying Lemma 5, without loss of generality, we shall write $\mathcal{E}_{g}^{T}[\cdot]$ simple as $\mathcal{E}_{g}[\cdot]$.

S. Peng [P1] showed that the $g$-expectation and the conditional $g$-expectation of a random variable $\xi$ preserve many of the basic properties of the mathematical expectation and conditional expectation (except linearity). For example,

Property 1 (1) $\mathcal{E}_{g}[\xi]=\mathcal{E}_{g}\left[\mathcal{E}_{g}\left[\xi \mid \mathcal{F}_{t}\right]\right], \quad \mathcal{E}_{g}[a]=a, \forall a \in R$

(2) $\mathcal{E}_{g}\left[\mathcal{E}_{g}\left[\xi \mid \mathcal{F}_{t}\right] \mid \mathcal{F}_{r}\right]=\mathcal{E}_{g}\left[\xi \mid \mathcal{F}_{r \wedge t}\right]$;

(3) $\mathcal{E}_{g}\left[\xi+\eta \mid \mathcal{F}_{t}\right]=\xi+\mathcal{E}_{g}\left[\eta \mid \mathcal{F}_{t}\right]$, if $\xi$ is $\mathcal{F}_{t}$-measurable.

The following examples show a relation between $g$-expectations and mathematical expectations:

Example 1 If $\xi \in L^{2}\left(\Omega, \mathcal{F}_{T}, P\right)$ and $g(y, z, t)=a(t)|z|$, here $a:=\{a(t)\}$ is a square integrable process bounded by $u$, then

(i) g-expectation $\mathcal{E}_{a}[\xi]$ :

$$
\mathcal{E}_{a}[\xi]= \begin{cases}E \xi, & a=0 \\ \inf _{Q \in \mathcal{P}} E_{Q}[\xi], & a<0 \\ \sup _{Q \in \mathcal{P}} E_{Q}[\xi], & a>0\end{cases}
$$

(ii) Conditional g-expectation:

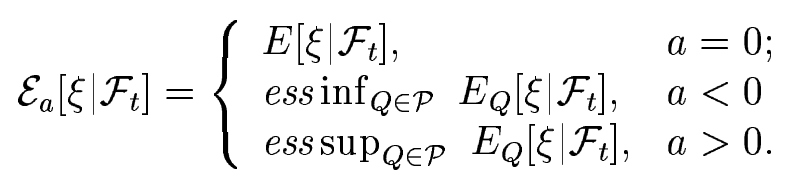


(iii) g-probability : $\forall A \in \mathcal{F}_{T}$,

$$
P_{a}(A)= \begin{cases}P(A), & a=0 \\ \inf _{Q \in \mathcal{P}} Q(A), & a<0 \\ \sup _{Q \in \mathcal{P}} Q(A), & a>0\end{cases}
$$

where $\mathcal{P}:=\left\{Q^{v}: \frac{d Q^{v}}{d P}:=e^{-\frac{1}{2} \int_{0}^{T}\left|v_{s}\right|^{2} d s+\int_{0}^{T} v_{s} d W_{s}},\left|v_{t}\right| \leq\left|a_{t}\right|\right.$, a.e. $\left.t \in[0, T]\right\}$.

Proof. We recall briefly the proof which can be found in [KQ, CP]. We just prove the claims (i),(ii) for $a \leq 0$, the rest can be proved in the same way.

If $a \leq 0$, for any $\xi \in L^{2}\left(\Omega, \mathcal{F}_{T}, P\right)$, set $(y, z):=\left(\mathcal{E}_{a}\left[\xi \mid \mathcal{F}_{t}\right], V_{t}(\xi, a)\right)$. By the definition of $\mathcal{E}_{g}[\cdot],\left(y_{t}, z_{t}\right)$ is the unique solution of the BSDE:

$$
y_{t}=\xi+\int_{t}^{T} a(s)\left|z_{s}\right| d s-\int_{t}^{T} z_{s} d W_{s}, \quad 0 \leq t \leq T .
$$

Set $a_{s}:=a(s) \operatorname{sgn} z_{s}$, then BSDE (5) can be rewritten as follows:

$$
y_{t}=\xi-\int_{t}^{T} z_{s} d \bar{W}_{s}
$$

where $\bar{W}_{t}=W_{t}-\int_{0}^{t} a_{s} d s$. By Girsanov's Theorem, $\left\{\bar{W}_{t}\right\}$ is a $Q$-Brownian motion, where $E\left[\frac{d Q}{d P} \mid \mathcal{F}_{T}\right]:=\mathrm{e}^{-\frac{1}{2} \int_{0}^{T} a_{s}^{2} d s+\int_{0}^{T} a_{s} d W_{s}}$. Taking conditional expectation $E_{Q}\left[\cdot \mid \mathcal{F}_{t}\right]$ on both sides of the BSDE (6), we get

$$
y_{t}=E_{Q}\left[\xi \mid \mathcal{F}_{t}\right] \geq \operatorname{ess} \inf _{Q \in \mathcal{P}} E_{Q}\left[\xi \mid \mathcal{F}_{t}\right]
$$

that is ,

$$
\mathcal{E}_{a}\left[\xi \mid \mathcal{F}_{t}\right] \geq \operatorname{ess} \inf _{Q \in \mathcal{P}} E_{Q}\left[\xi \mid \mathcal{F}_{t}\right]
$$

On the other hand, let $\left\{\theta_{t}\right\}$ be any process bounded by $\left\{\left|a_{t}\right|\right\}$, i.e. $\left|\theta_{t}\right| \leq\left|a_{t}\right|$. Applying Lemma 1 , the following BSDE has a unique solution $\left(y^{\theta}, z^{\theta}\right)$,

$$
y_{t}^{\theta}=\xi+\int_{t}^{T} \theta_{s} z_{s} d s-\int_{t}^{T} z_{s} d W_{s}, \quad 0 \leq t \leq T .
$$

Let $E\left[\frac{d Q^{\theta}}{d P} \mid \mathcal{F}_{T}\right]:=\mathrm{e}^{-\frac{1}{2} \int_{0}^{T} \theta_{s}^{2} d s+\int_{0}^{T} \theta_{s} d W_{s}}$. Solving BSDE (7), we have

$$
y_{t}^{\theta}=E_{Q^{\theta}}\left[\xi \mid \mathcal{F}_{t}\right]
$$

$\mathrm{RR} \mathrm{n}^{\circ} 4284$ 
Since $a(t) \leq 0$, thus $\theta_{t} z \geq a(t)|z|$, for all $(z, t) \in \mathbb{R} \times[0, T]$, comparing (5) with (7) and applying the comparison theorem ([P1], Theorem 35.3), we obtain

$$
E_{Q^{\theta}}\left[\xi \mid \mathcal{F}_{t}\right]=y_{t}^{\theta} \geq y_{t}
$$

Consequently

$$
\operatorname{ess} \inf _{Q \in \mathcal{P}} E_{Q}\left[\xi \mid \mathcal{F}_{t}\right] \geq \mathcal{E}_{a}\left[\xi \mid \mathcal{F}_{t}\right]
$$

Hence

$$
\mathcal{E}_{a}\left[\xi \mid \mathcal{F}_{t}\right]=\operatorname{ess} \inf _{Q \in \mathcal{P}} E_{Q}\left[\xi \mid \mathcal{F}_{t}\right] .
$$

This with Remark 2 (1) completes the proof of (ii) for $a \leq 0$. In particular, let $t=0$, we obtain (i) for $a \leq 0$.

Definition 2 (1) The random variable $\xi$ and $\eta$ are called comonotonic, if

$$
\left[\xi(\omega)-\xi\left(\omega^{\prime}\right)\right]\left[\eta(\omega)-\eta\left(\omega^{\prime}\right)\right] \geq 0, \forall \omega, \omega^{\prime} \in \Omega .
$$

(2) A real function $F$ defined on $L^{2}\left(\Omega, \mathcal{F}_{T}, P\right)$ is called comonotonic additive, if

$$
F(\xi+\eta)=F(\xi)+F(\eta) \text {, whenever } \xi \text { and } \eta \text { are comonotonic. }
$$

(3) A real valued set function $V: \mathcal{F}_{T} \rightarrow[0,1]$ is called a capacity if

(i) $V(\emptyset)=0, \quad V(\Omega)=1$

(ii) $V(A) \leq V(B)$ for any $A \subset B$.

(4) Choquet integral: Let $V$ be a capacity, for any $\xi \in L^{2}\left(\Omega, \mathcal{F}_{T}, P\right)$, the functional $F(\xi)$ defined by

$$
F(\xi):=\int_{-\infty}^{0}(V(\xi \geq t)-1) d t+\int_{0}^{\infty} V(\xi \geq t) d t
$$

is called the Choquet integral of $\xi$ with respect to $V$.

\section{Main Result.}

Let $H$ be the set of all $\mathcal{F}_{T}$-measurable random variables $X_{T} \in L^{2}\left(\Omega, \mathcal{F}_{T}, P\right)$ which satisfy that there exist $\sigma, b$ satisfying Assumption A, such that $X_{T}$ is the value of the solution $\left\{X_{t}\right\}$ of the following forward stochastic differential equation (SDE) at time $T$,

$$
\left\{\begin{array}{l}
d X_{s}=b\left(s, X_{s}\right) d s+\sigma\left(s, X_{s}\right) d W_{s}, \quad 0 \leq s \leq T \\
X_{0}=x \in \mathbb{R} .
\end{array}\right.
$$

We denote by $1_{A}$ the indicator function of the event $A$ and denote $V(A):=\mathcal{E}_{g}\left[1_{A}\right]$. 
Theorem 1 Suppose that g satisfy (H1)(H2) and (H3).

$\mathcal{E}_{g}[\xi]$ can be represented as a Choquet integral for any $\xi \in H$, that is,

$$
\mathcal{E}_{g}[\xi]=\int_{-\infty}^{0}[V(\xi \geq t)-1] d t+\int_{0}^{\infty} V(\xi \geq t) d t
$$

if and only if there exist two continuous functions a $b$ such that $g$ is of the form

$$
g(y, z, t):=a(t)|z|+b(t) z .
$$

We shall prove this theorem in the next sections.

\section{Necessary condition for integral representation.}

For a given capacity $V$, Choquet (1955) defined an integral which is called the Choquet integral. Dellacherie (1970) and Schmeidler (1989) for the general case showed that $F(\cdot)$, the operator of Choquet integral defined in Definiton 2 (4), is of comonotonic additivity, that is

$$
F(\xi+\eta)=F(\xi)+F(\eta) \text {, whenever } \xi, \eta \text { are comonotonic. }
$$

We use here Dellacherie and Schmeidler's results to investigate a necessary condition for $\mathcal{E}_{g}[\cdot]$ to be represented by a Choquet integral. We need the following lemmas. Lemma 6 extends the corresponding result in [BCHMP, CE].

Lemma 6 Suppose that $\left\{X_{t}\right\}$ is of the form

$$
X_{t}=x+\int_{0}^{t} a_{s} d s+\int_{0}^{t} b_{s} d W_{s}, \quad 0 \leq t \leq T,
$$

where $\left\{a_{t}\right\}$ and $\left\{b_{t}\right\}$ are two continuous bounded processes. Then

(i) $\mathcal{E}_{g}\left[X_{\tau} \mid \mathcal{F}_{t}\right] \rightarrow X_{t}, \quad V_{t}\left(X_{\tau}, g\right) \rightarrow b_{t}$, as $\tau \rightarrow t$

(ii) $\lim _{\tau \rightarrow t^{+}} \frac{\mathcal{E}_{g}\left[\xi_{\tau} \mid \mathcal{F}_{t}\right]-E\left[X_{\tau} \mid \mathcal{F}_{t}\right]}{\tau-t}=g\left(X_{t}, b_{t}, t\right)$,

where the limits are in the sense of $L^{2}\left(\Omega, \mathcal{F}_{t}, P\right)$.

Proof. Because $a$ and $b$ are bounded, for any $\tau \in[0, T], X_{\tau} \in L^{2}\left(\Omega, \mathcal{F}_{T}, P\right)$. Let $\left(y_{t}, z_{t}\right):=$ $\left(\mathcal{E}_{g}\left[X_{\tau} \mid \mathcal{F}_{t}\right], V_{t}\left(X_{\tau}, g\right)\right)$. Thanks to assumption (H3), $\left(y_{t}, z_{t}\right)$ is the solution of the BSDE:

$$
y_{t}=X_{\tau}+\int_{t}^{T} g\left(y_{s}, z_{s}, s\right) d s-\int_{t}^{T} z_{s} d W_{s}
$$

$\mathrm{RR} \mathrm{n}^{\circ} 4284$ 
with

$$
z_{t} \equiv 0 \text {, a.e. } t \in(\tau, T] .
$$

Hence the above BSDE can be written as

$$
y_{t}=X_{\tau}+\int_{t}^{\tau} g\left(y_{s}, z_{s}, s\right) d s-\int_{t}^{\tau} z_{s} d W_{s} .
$$

Applying Lemma 2, there exists a constant $c>0$ such that

$$
E\left[\int_{t}^{\tau}\left|z_{s}\right|^{2} d s \mid \mathcal{F}_{t}\right] \leq c E\left[\left|X_{\tau}\right|^{2} \mid \mathcal{F}_{t}\right] .
$$

Taking the conditional expectation $E\left[\cdot \mid \mathcal{F}_{t}\right]$ on both sides of $\operatorname{BSDE}(9)$, we obtain

$$
\begin{aligned}
\left|\mathcal{E}_{g}\left[X_{\tau} \mid \mathcal{F}_{t}\right]-E\left[X_{\tau} \mid \mathcal{F}_{t}\right]\right|^{2} & =\left|y_{t}-E\left[X_{\tau} \mid \mathcal{F}_{t}\right]\right|^{2} \\
& =\left|E\left[\int_{t}^{\tau} g\left(y_{s}, z_{s}, s\right) d s \mid \mathcal{F}_{t}\right]\right|^{2} \\
& \leq u^{2}(\tau-t) E\left[\int_{t}^{\tau}\left|z_{s}\right|^{2} d s \mid \mathcal{F}_{t}\right] \\
& \leq c u^{2}(\tau-t) E\left[\sup _{T>s>0}\left|X_{s}\right|^{2} \mid \mathcal{F}_{t}\right] \\
& \rightarrow 0 \text { as } \tau \rightarrow t \text { in } L^{1}\left(\Omega, \mathcal{F}_{t}, P\right),
\end{aligned}
$$

which implies $\mathcal{E}_{g}\left[X_{\tau} \mid \mathcal{F}_{t}\right] \rightarrow E\left[X_{\tau} \mid \mathcal{F}_{t}\right]$ as $\tau \rightarrow t$ in $L^{2}\left(\Omega, \mathcal{F}_{T}, P\right)$.

On the other hand, from (8),

$$
X_{\tau}=X_{t}+\int_{t}^{\tau} a_{s} d s+\int_{t}^{\tau} b_{s} d W_{s}
$$

thus

$$
E\left[X_{\tau} \mid \mathcal{F}_{t}\right]-X_{t}=E\left[\int_{t}^{\tau} a_{s} d s \mid \mathcal{F}_{t}\right] \rightarrow 0 \text { as } \tau \rightarrow t \text { in } L^{2}\left(\Omega, \mathcal{F}_{t}, P\right) .
$$

Hence

$$
\mathcal{E}_{g}\left[X_{\tau} \mid \mathcal{F}_{t}\right] \rightarrow X_{t} \text { as } \tau \rightarrow t
$$

So

$$
<\mathcal{E}_{g}\left[X_{\tau} \mid \mathcal{F}_{t}\right], W_{t}>\rightarrow<X_{t}, W_{t}>\text {, as } \tau \rightarrow t
$$

But

$$
<\mathcal{E}_{g}\left[X_{\tau} \mid \mathcal{F}_{t}\right], W_{t}>=\int_{0}^{t} V_{s}\left(X_{\tau}, g\right) d s, \quad<X_{t}, W_{t}>=\int_{0}^{t} b_{s} d s .
$$

Hence

$$
V_{t}\left(X_{\tau}, g\right) \rightarrow b_{t}, \text { a.e., as } \tau \rightarrow t \text {, in } L^{2}\left(\Omega, \mathcal{F}_{t}, P\right) .
$$

Here $\langle X, Y\rangle$ is the covariance process associated to the semi-martingales $X$ and $Y$. The proof of (i) is thus complete. 
Applying (i) and the continuity of $g$, we get

$$
\begin{aligned}
\frac{\mathcal{E}_{g}\left[X_{\tau} \mid \mathcal{F}_{t}\right]-E\left[X_{\tau} \mid \mathcal{F}_{t}\right]}{\tau-t} & =\frac{1}{\tau-t} E\left[\int_{t}^{\tau} g\left(\mathcal{E}_{g}\left[X_{\tau} \mid \mathcal{F}_{s}\right], V_{s}\left(X_{\tau}, g\right), s\right) d s \mid \mathcal{F}_{t}\right] \\
& \rightarrow g\left(X_{t}, b_{t}, t\right), \quad \text { as } \tau \rightarrow t
\end{aligned}
$$

in $L^{2}\left(\Omega, \mathcal{F}_{t}, P\right)$. The proof of (ii) is thus complete.

Lemma 7 If $\mathcal{E}_{g}[\cdot]$ is comonotonic additive in $H$, that is

$$
\mathcal{E}_{g}[\xi+\eta]=\mathcal{E}_{g}[\xi]+\mathcal{E}_{g}[\eta], \text { whenever } \xi \text { and } \eta \in H \text { are comonotonic, }
$$

then there exist two continuous functions $a:=\{a(t)\}, b:=\{b(t)\}$ such that $g$ is of the form:

$$
g(y, z, t)=a(t) z^{+}-b(t) z^{-},
$$

where $z^{+}:=\max \{z, 0\}$ and $z^{-}:=\min \{z, 0\}$.

Proof. For each $i=1,2$, let us choose $\left(y_{i}, z_{i}, t\right) \in \mathbb{R}^{2} \times[0, T]$ such that $z_{1} z_{2} \geq 0$.

For any $\tau \in[t, T]$, let $\xi=y_{1}+z_{1}\left(W_{\tau}-W_{t}\right)$ and $\eta=y_{2}+z_{2}\left(W_{\tau}-W_{t}\right)$. Obviously $\xi$ and $\eta$ are comonotonic. Note that

$$
\mathcal{E}_{g}\left[\xi \mid \mathcal{F}_{t}\right]=\mathcal{E}_{g}[\xi], \quad \mathcal{E}_{g}\left[\eta \mid \mathcal{F}_{t}\right]=\mathcal{E}_{g}[\eta], \quad \mathcal{E}_{g}\left[\xi+\eta \mid \mathcal{F}_{t}\right]=\mathcal{E}_{g}[\xi+\eta]
$$

From (10), we have

$$
\frac{\mathcal{E}_{g}\left[\xi+\eta \mid \mathcal{F}_{t}\right]-E\left[\xi+\eta \mid \mathcal{F}_{t}\right]}{\tau-t}=\frac{\mathcal{E}_{g}\left[\xi \mid \mathcal{F}_{t}\right]-E\left[\xi \mid \mathcal{F}_{t}\right]}{\tau-t}+\frac{\mathcal{E}_{g}\left[\eta \mid \mathcal{F}_{t}\right]-E\left[\eta \mid \mathcal{F}_{t}\right]}{\tau-t}
$$

Applying Lemma 6 (ii) and the fact that

$$
V_{t}(\xi+\eta, g) \rightarrow y_{1}+y_{2}, V_{t}(\xi, g) \rightarrow y_{1}
$$

and

$$
V_{t}(\eta, g) \rightarrow y_{2}, \text { as } \tau \rightarrow t
$$

we obtain

$$
g\left(y_{1}+y_{2}, z_{1}+z_{2}, t\right)=g\left(y_{1}, z_{1}, t\right)+g\left(y_{2}, z_{2}, t\right), \forall z_{1} z_{2} \geq 0,
$$

this with (H3) imply that there exist two continuous functions $a$ and $b$ bounded by $\{u\}$ such that

$$
g(y, z, t,)=a(t) z^{+}-b(t) z^{-} .
$$

Using the above method, we can easily get,

Corollary 1 The operator of $g$-expectation $\mathcal{E}_{g}[\cdot]$ is linear iff $g$ is linear.

$\mathrm{RR} \mathrm{n}^{\circ} 4284$ 
In [P1], Peng showed that if $g$ is convex then $\mathcal{E}_{g}[\cdot]$ is convex. Hence,

Corollary $2 \mathcal{E}_{g}[\cdot]$ is convex, if and only if $g$ is convex.

Using the above lemmas, we can give a necessary condition for the $g$-expectation to be represented by a Choquet integral.

Theorem 2 If $\mathcal{E}_{g}[\cdot]$ can be represented by a Choquet integral, then there exist two continuous functions $\alpha$ and $\beta$ such that $g$ is of the form

$$
g(y, z, t)=\alpha(t)|z|+\beta(t) z .
$$

Proof. If $\mathcal{E}_{g}[\cdot]$ can be represented by a Choquet integral, by Dellacherie's Theorem in [De, 1970] ( for the general case, see Schmeidler [S]), then $\mathcal{E}_{g}[\cdot]$ satisfies comonotonic additivity, that is $\mathcal{E}_{g}[\xi+\eta]=\mathcal{E}_{g}[\xi]+\mathcal{E}_{g}[\eta]$ whenever $\xi$ and $\eta$ are comonotonic. Applying Lemma 7, $g$ is of the form: $g(y, z, t)=a(t) z^{+}-b(t) z^{-}$. Set $\alpha(t):=\frac{a(t)-b(t)}{2}$ and $\beta(t):=$ $\frac{a(t)+b(t))}{2}$, we complete the proof.

Remark 4 If $g$ is of the form (15), then $y_{t}:=\mathcal{E}_{g}\left[\xi \mid \mathcal{F}_{t}\right]$ is the solution of the BSDE:

$$
y_{t}=\xi+\int_{t}^{T}\left[\alpha(s)\left|z_{s}\right|+\beta(s) z_{s}\right] d s-\int_{t}^{T} z_{s} d W_{s},
$$

which can be rewritten as

$$
y_{t}=\xi+\int_{t}^{T} \alpha(s)\left|z_{s}\right| d s-\int_{t}^{T} z_{s} d \bar{W}_{s},
$$

where $\bar{W}_{t}:=W_{t}-\int_{0}^{t} \beta(s) d s$, is a $Q$-Brownian motion under $Q$ defined by

$$
\frac{d Q}{d P}=\exp \left\{-\frac{1}{2} \int_{0}^{T} \beta^{2}(s) d s+\int_{0}^{T} \beta(s) d W_{s}\right\} .
$$

Thus, without loss of generality, in the rest of this paper, we assume $\beta \equiv 0$. Otherwise we can consider our problem in the probability space $(\Omega, \mathcal{F}, Q)$.

\section{Sufficient condition for integral representation}

In this section, we will prove that the necessary condition in Theorem 2 is also sufficient. To do so, we need the following lemmas:

For any $X_{T} \in H$, let $X_{T}$ be the value of $\left\{X_{t}\right\}$, the solution of the following SDE, at time $T$ :

$$
\left\{\begin{array}{l}
d X_{s}=b\left(s, X_{s}\right) d s+\sigma\left(s, X_{s}\right) d W_{s}, 0 \leq s \leq T \\
X_{0}=x, x \in R
\end{array}\right.
$$

where $b, \sigma$ satisfy Assumption A. 
Lemma 8 Suppose that $\Phi$ and $\Psi$ are two monotonic functions such that $\Phi\left(X_{T}\right)$ and $\eta=\Psi\left(X_{T}\right) \in L^{2}(\Omega, \mathcal{F}, P)$, and let $\left(y^{\Phi}, z^{\Phi}\right)$ and $\left(y^{\Psi}, z^{\Psi}\right)$ be the solutions of the BSDE:

$$
y_{t}=\xi+\int_{t}^{T} g\left(y_{s}, z_{s}, s\right) d s-\int_{t}^{T} z_{s} d W_{s}
$$

with terminal values $\xi=\Phi\left(X_{T}\right)$ and $\xi=\Psi\left(X_{T}\right)$, respectively. If $b, \sigma, \Phi, \Psi$ and $g$ are assumed to be $C^{3}$, then

$$
z_{t}^{\Phi} z_{t}^{\Psi} \geq 0, \quad \text { a.e. } t \in[0, T] .
$$

Proof. Let $\left\{X_{s}^{t, x}\right\}$ be the solution of the SDE:

$$
\left\{\begin{array}{l}
d X_{s}^{t, x}=b\left(s, X_{s}^{t, x}\right) d t+\sigma\left(s, X_{s}^{t, x}\right) d W_{s}, \\
X_{t}=x, \quad s \in[t, T] .
\end{array}\right.
$$

and let $\left(y_{s}^{t, x, \Phi}, z_{s}^{t, x, \Phi}\right)$ and $\left(y_{s}^{t, x, \Psi}, z_{s}^{t, x, \Psi}\right)$ be the solutions of the following BSDE corresponding to $\xi=\Phi\left(X_{T}^{t . x}\right), g=g_{1}$ and $\xi=\Psi\left(X_{T}^{t, x}\right), g=g_{2}$, respectively:

$$
y_{t}=\xi+\int_{t}^{T} g\left(y_{s}, z_{s}, s\right) d s-\int_{t}^{T} z_{s} d W_{s}
$$

then, obviously, $X_{T}=X_{T}^{0, x}$ and $z_{s}^{\Phi}=z_{s}^{0, x, \Phi}, z_{s}^{\Psi}=z_{s}^{0, x, \Psi}$.

But by Lemma 3(ii),

$$
\left\{\begin{array}{l}
z_{s}^{t, x, \Phi}=\sigma\left(s, X_{s}^{t, x}\right) \partial_{x} u\left(s, X_{s}^{t, x}\right), \text { a.e. } s \in[0, T] \\
z_{s}^{t, x, \Psi}=\sigma\left(s, X_{s}^{t, x}\right) \partial_{x} v\left(s, X_{s}^{t, x}\right), \text { a.e. } s \in[0, T]
\end{array}\right.
$$

where $u(t, x):=y_{t}^{t, x, \Phi}$ and $v(t, x):=y_{t}^{t, x, \Psi}$. Thus, if $t=0$, it follows

$$
\begin{aligned}
z_{s}^{\Phi} z_{s}^{\Psi} & =z_{s}^{0, x, \Phi} z_{s}^{0, x, \Psi} \\
& =\sigma\left(s, X_{s}\right) \partial_{x} u\left(s, X_{s}\right) \sigma\left(s, X_{s}\right) \partial_{x} v\left(s, X_{s}\right) \\
& =\sigma^{2}\left(s, X_{s}\right) \partial_{x} u\left(s, X_{s}\right) \partial_{x} v\left(s, X_{s}\right), \text { a.e. } s \in[0, T] .
\end{aligned}
$$

If $\Phi, \Psi$ are monotonic, applying the Comparison Theorem for SDE and BSDE, it is easy to check that $u(t, x)$ and $v(t, x)$ are monotonic, from (19). The proof is complete.

Remark 5 By Lemma 2, the assumption that $b, \sigma, g, \Phi$ and $\Psi$ are $C^{3}$ in Lemma 8 is not necessary. Indeed, if there exist $b^{\epsilon}, \sigma^{\epsilon}, g^{\epsilon}, \Phi_{\epsilon}$ and $\Psi_{\epsilon}$ such that $b^{\epsilon}, \sigma^{\epsilon}, g^{\epsilon}, \Phi_{\epsilon}, \Psi_{\epsilon}$ satisfy the assumptions of Lemma 8 and converge to $b, \sigma, g, \Phi, \Psi$ as $\epsilon \rightarrow 0$, then by Lemma 2, the result of Lemma 8 is still true.

$\mathrm{RR} \mathrm{n}^{\circ} 4284$ 
Let us now consider the case where $\Phi$ and $\Psi$ are indicator fonctions:

Suppose that $\left\{X_{t}\right\}$ is the solution of $\operatorname{SDE}(16)$. For any $b<c$, set $B=\left\{X_{T} \geq b\right\}$ and $C=\left\{X_{T} \geq c\right\}$ and let $\left(y^{B}, z^{B}\right)$ and $\left(y^{C}, z^{C}\right)$ be the solutions of the following BSDE corresponding to $1_{B}$ and $1_{C}$ respectively:

$$
y_{t}=\xi+\int_{t}^{T} g\left(y_{s}, z_{s}, s\right) d s-\int_{t}^{T} z_{s} d W_{s}
$$

Obviously, $C \subset B$, and we obtain,

Lemma 9 Using the above notation, suppose that $b, \sigma, g$ satisfy the assumptions of Lemma 8, then

$$
z_{t}^{C} z_{t}^{B} \geq 0, \quad \text { a.e. } t \in[0, T] .
$$

Proof. First, let us construct $C^{3}$ functions which converge to indicator functions.

Indeed, for any $n=1,2, \cdots$, we denote by $\Phi_{n}(x, b)$ and $\Phi_{n}(x, c)$ :

$$
\Phi_{n}(x, b):=e^{-n d^{(b)}(x)}, \quad \Phi_{n}(x, c):=e^{-n d^{(c)}(x)},
$$

where

$$
d^{(b)}(x)=\left\{\begin{array}{ll}
(b-x)^{3}, & x<b, \\
0, & x \geq b ;
\end{array} ; d^{(c)}(x)= \begin{cases}(c-x)^{3}, & x<c, \\
0, & x \geq c .\end{cases}\right.
$$

It is easy to check that for each $n \geq 1, \Phi_{n}(x, b), \Phi_{n}(x, c) \in C^{3}$ and

$$
\Phi_{n}(x, a) \rightarrow 1_{(x \leq a)}, \quad \Phi_{n}(x, b) \rightarrow 1_{(x \geq b)}, \quad \Phi_{n}(x, c) \rightarrow 1_{(x \geq c)} \text { as } n \rightarrow \infty .
$$

Let us now prove (20):

Let $\left(y^{n, b}, z^{n, b}\right)$ and $\left(y^{n, c}, z^{n, c}\right)$ be the solutions of the following BSDE corresponding to $\xi=\Phi_{n}\left(X_{T}, b\right)$ and $\xi=\Phi_{n}\left(X_{T}, c\right)$ respectively

$$
y_{t}=\xi+\int_{t}^{T} g\left(y_{s}, z_{s}, z\right) d s-\int_{t}^{T} z_{s} d W_{s}
$$

Since $\Phi_{n}(x, b)$ and $\Phi_{n}(x, c)$ are monotonic, applying Lemma 8 , we have

$$
z_{s}^{n, b} z_{s}^{n, c} \geq 0, \quad \text { a.e. } s \in[0, T] .
$$

Note that $\Phi_{n}\left(X_{T}, b\right) \rightarrow 1_{B}$ and $\Phi_{n}\left(X_{T}, c\right) \rightarrow 1_{C}$ as $n \rightarrow \infty$ in $L^{2}(\Omega, \mathcal{F}, P)$, by Lemma 2 $z^{n, b} \rightarrow z^{B}$ and $z^{n, c} \rightarrow z^{C}$ as $n \rightarrow \infty$ in $L^{2}(0, T)$. The proof is complete.

Remark 6 In Lemma 9, if we replace $X_{T}$ by $\Phi\left(X_{T}\right)$, where $\Phi$ is monotonic, then the corresponding result of Lemma 9 is still true. 
We now assume that $g$ is of the form:

$$
g(y, z, t)=a(t)|z|
$$

where $a$ is a continuous function bounded by $\mu$.

Lemma 10 Suppose that $\xi \in H$ and $\alpha_{1} \leq \alpha_{2} \leq \cdots \alpha_{n}$ is a sequence in $R$. Let $A_{i}:=\{\omega$ : $\left.\xi(\omega) \geq \alpha_{i}\right\}, i=1,2, \cdots, n$. Then for any positive sequence $\left\{b_{i}\right\}_{i=1}^{n}$,

$$
\mathcal{E}_{a}\left[\sum_{i=1}^{n} b_{i} 1_{A_{i}} \mid \mathcal{F}_{t}\right]=\sum_{i=1}^{n} b_{i} \mathcal{E}_{a}\left[1_{A_{i}} \mid \mathcal{F}_{t}\right], t \in[0, T]
$$

In particular,

$$
\mathcal{E}_{a}\left[\sum_{i=1}^{n} b_{i} 1_{A_{i}}\right]=\sum_{i=1}^{n} b_{i} \mathcal{E}_{a}\left[1_{A_{i}}\right]
$$

Proof. For any $\epsilon>0$, the function $g_{\epsilon}$ defined by $g_{\epsilon}(y, z, t):=a(t) \sqrt{|z|^{2}+\epsilon}$, is differentiable with respect to $z$ and its derivative is uniformly bounded.

Let $\left(y_{t}^{i, \epsilon}, z_{t}^{i, \epsilon}\right)$ be the solution of the BSDE

$$
\left.y_{t}=1_{A_{i}}+\int_{t}^{T} a(s) \sqrt{\left(\left|z_{s}\right|^{2}+\epsilon\right.}\right) d s-\int_{t}^{T} z_{s} d W_{s}, i=1.2, \cdots, n .
$$

Applying Lemma 2 again, $\left(y_{t}^{i, \epsilon}, z_{t}^{i, \epsilon}\right) \rightarrow\left(y^{i}, z^{i}\right)$ as $\epsilon \rightarrow 0$ in $L^{2}(0, T) \times L^{2}(0, T)$, where $\left(y^{i}, z^{i}\right)$ are the solutions of the BSDE:

$$
y_{t}^{i}=1_{A_{i}}+\int_{t}^{T} a(s)\left|z_{s}^{i}\right| d s-\int_{t}^{T} z_{s}^{i} d W_{s}, i=1.2, \cdots, n .
$$

Lemma 9 implies that for any $i, j=1,2, \cdots, n$,

$$
z_{t}^{i, \epsilon} z_{t}^{j, \epsilon} \geq 0
$$

Hence,

$$
z_{t}^{i} z_{t}^{j} \geq 0, \text { a.e. } t \in[0, T], \quad i, j=1,2, \cdots, n,
$$

which implies

$$
\left|\sum_{i=1}^{n} z_{t}^{i}\right|=\sum_{i=1}^{n}\left|z_{t}^{i}\right| \quad \text { a.e.t } \in[0, T] .
$$

Multiplying by $b_{i}$ both sides of BSDE (23), adding from $i=1$ to $n$, and applying (24), we obtain

$$
\sum_{i=1}^{n} b_{i} y_{t}^{i}=\sum_{i=1}^{n} b_{i} 1_{A_{i}}+\int_{t}^{T} a(s)\left|\sum_{i=1}^{n} b_{i} z_{s}^{i}\right| d s-\int_{t}^{T} \sum_{i=1}^{n} b_{i} z_{s}^{i} d W_{s}
$$

$\mathrm{RR} \mathrm{n}^{\circ} 4284$ 
which means that $\left(\sum_{i=1}^{n} b_{i} y_{t}^{i}, \sum_{i=1}^{n} b_{i} z_{t}^{i}\right)$ is the solution of the BSDE:

$$
y_{t}=\sum_{i=1}^{n} b_{i} 1_{A_{i}}+\int_{t}^{T} a(s)\left|z_{s}\right| d s-\int_{t}^{T} z_{s} d W_{s} .
$$

By the uniqueness of the solution of the BSDE,

$$
\mathcal{E}_{a}\left[\sum_{i=1}^{n} b_{i} 1_{A_{i}} \mid \mathcal{F}_{t}\right]=\sum_{i=1}^{n} b_{i} y_{t}^{i}=\sum_{i=1}^{n} b_{i} \mathcal{E}_{a}\left[1_{A_{i}} \mid \mathcal{F}_{t}\right], t \in[0, T]
$$

In particular, taking $t=0$, we obtain (22)

Using the lemmas above, we can show our main theorem in this section.

Theorem 3 Assume $g$ is of the form (21), then, for any $\xi \in H, \mathcal{E}_{a}[\xi]$ can be represented as a Choquet integral, i.e. $\mathcal{E}_{a}[\xi]$ can be represented in the form $\left({ }^{*}\right)$.

Proof. The proof is divided into two steps:

Step 1: Assume that $\xi$ is bounded by $N$, i.e. $|\xi| \leq N$.

First, assume $\xi \geq 0$.

Set

$$
\xi_{-}^{(n)}:=\sum_{i=1}^{2^{n}} \frac{i N}{2^{n}} 1_{\left(\frac{i N}{2^{n}} \leq \xi<\frac{(i+1) N}{2^{n}}\right)} ; \quad \xi_{+}^{(n)}:=\sum_{i=1}^{2^{n}} \frac{(i+1) N}{2^{n}} 1_{\left(\frac{i N}{2^{n}} \leq \xi<\frac{(i+1) N}{2^{n}}\right)},
$$

then $\xi_{-}^{(n)} \leq \xi \leq \xi_{+}^{(n)}, \quad \xi_{-}^{(n)} \rightarrow \xi, \quad \xi_{+}^{(n)} \rightarrow \xi$ as $n \rightarrow \infty$ in $L^{2}\left(\Omega, \mathcal{F}_{T}, P\right)$ and

$$
\lim _{n \rightarrow \infty} \mathcal{E}_{a}\left[\xi_{-}^{(n)}\right]=\lim _{n \rightarrow \infty} \mathcal{E}_{a}\left[\xi_{+}^{(n)}\right]=\mathcal{E}_{a}[\xi] .
$$

Note that it is easy to rewrite $\xi_{-}^{(n)}$ and $\xi_{+}^{(n)}$ as

$$
\xi_{-}^{(n)}=\sum_{i=1}^{2^{n}} \frac{N}{2^{n}} 1_{\left(\xi \geq \frac{i N}{2^{n}}\right)}, \quad \xi_{+}^{(n)}=\sum_{i=1}^{2^{n}} \frac{N}{2^{n}} 1_{\left(\xi \geq \frac{(i-1) N}{2^{n}}\right)} .
$$

Applying (22), remembering the definition of $P_{a}(A)=\mathcal{E}_{a}\left[1_{A}\right]$, we have

$$
\mathcal{E}_{a}\left[\xi_{-}^{(n)}\right]=\sum_{i=1}^{2^{n}} \frac{N}{2^{n}} P_{a}\left(\xi \geq \frac{i N}{2^{n}}\right), \quad \mathcal{E}_{a}\left[\xi_{+}^{(n)}\right]=\sum_{i=1}^{2^{n}} \frac{N}{2^{n}} P_{a}\left(\xi \geq \frac{(i-1) N}{2^{n}}\right) .
$$

But

$$
\sum_{i=1}^{2^{n}} \frac{N}{2^{n}} P_{a}\left(\xi \geq \frac{i N}{2^{n}}\right) \leq \int_{0}^{N} P_{a}(\xi \geq t) d t \leq \sum_{i=1}^{2^{n}} \frac{N}{2^{n}} P_{a}\left(\xi \geq \frac{(i-1) N}{2^{n}}\right) .
$$


Hence,

$$
\mathcal{E}_{a}\left[\xi_{-}^{(n)}\right] \leq \int_{0}^{N} P_{a}(\xi \geq t) d t \leq \mathcal{E}_{a}\left[\xi_{+}^{(n)}\right]
$$

Let $n \rightarrow \infty$,

$$
\mathcal{E}_{a}[\xi]=\int_{0}^{N} P_{a}(\xi \geq t) d t .
$$

Thus $\mathcal{E}_{a}[\xi]$ can be represented by the Choquet integral.

Second, if $\xi$ is not positive, let $\bar{\xi}=\xi+N$, then $0 \leq \bar{\xi} \leq 2 N$, Applying (25), we have

$$
\mathcal{E}_{a}[\bar{\xi}]=\int_{0}^{2 N} P_{a}(\bar{\xi} \geq t) d t=\int_{-N}^{N} P_{a}(\xi \geq t) d t
$$

By Property 1(3), we have

$$
\mathcal{E}_{a}[\bar{\xi}]=\mathcal{E}_{a}[\xi+N]=\mathcal{E}_{a}[\xi]+N
$$

Consequently,

$$
\mathcal{E}_{a}[\xi]=\mathcal{E}_{a}[\bar{\xi}]-N=\int_{-N}^{0}\left[P_{a}(\xi \geq t)-1\right] d t+\int_{0}^{N} P_{a}(\xi \geq t) d t .
$$

Step 2: Let $\Phi(x)=x \vee(-N) \wedge N$, then $\Phi$ is monotonic, and for any $\xi \in H$, $\xi^{N}:=\Phi(\xi)$ is bounded by $N$. By Step $1, \mathcal{E}_{a}\left[\xi^{N}\right]$ is of the form (26). Let $N \rightarrow \infty$ and note that $\lim _{N \rightarrow \infty} \mathcal{E}_{a}\left[\xi^{N}\right]=\mathcal{E}_{a}[\xi]$, we obtain

$$
\mathcal{E}_{a}[\xi]=\int_{-\infty}^{0}\left(P_{a}(\xi \geq t)-1\right) d t+\int_{0}^{\infty} P_{a}(\xi \geq t) d t
$$

Acknowledgments: The work was done while Chen was visiting INRIA in 1999, whose hospitality he deeply appreciated.

The authors have benefited from useful discussions with Shige Peng, Larry Epstein, Nicole El Karoui and J.P. Lepeltier.

\section{References}

[B] R. Buckdhan, Backward stochastic differential equations driven by a martingale, Preprint, 1993.

[BCHMP] P. Briand F. Coquet, Y. Hu , J. Memin, S.Peng, A converse comparison theorem for $B S D E s$ and related properties.

[C1] Z.Chen, A comonotonic theorem of BSDEs and its applications. Preprint, 2001.

$\mathrm{RR} \mathrm{n}^{\circ} 4284$ 
[C2] Z.Chen, A property of BSDEs, C.R. Acad. Sci. Paris, 326, P. 483-488.

[CP] Z. Chen, S.Peng, A downcrossing inequality and its application, Stat. and Proba. Letters, 1999.

[CE] Z.Chen, L. Epstein, Ambiguity, risk and asset return in continuous time, Preprint, 1999 .

[Ch] G. Choquet, Theory of capacities, Ann. Inst. Fourier (Grenoble)5, 131-295, 1955.

[D] R. Darling, Constructing gamma martingales with prescribed limits, using BSDEs, Ann. Prob., 23, 1234-1261, 1995.

[De] C. Dellacherie, Quelques commentaires sur les prolongements de capacités, Séminaire Prob. V, Strasbourg, Lecture Note in Math., vol. 191, Springer-Verlag, Berlin and Now York, 1970.

[KPQ] El. Karoui, S.Peng and M. Quenez,BSDEs in finance, Math. finance, 1997, No. 1, 1-71.

[KQ] El. Karoui and M. Quenez, Dynamic programming and pricing contingent claims incomplete markets, SIAM J. Control Optim. 33, 1995.

[LM] J.P. Lepeltier, J.S. Martin, BSDEs with continuous coefficients, Statistics \& Proba. Letters, 32, 1997.

[H] Y, Hu, Probabilistic interpretation for a systems of quasi elliptic PDE with Neumann boundary conditions, Stoch. Prob. and Appli. , 48, 107-121, 1993.

[HL] S.Hamadene and J.P. Lepeltier, Zero-sum stochastic differential games and BSDEs. Stoch. and Stoch. Report, 54, 221-231, 1995.

[PP1] E. Pardoux and S. Peng, Adapted Solution of a Backward Stochastic Differential Equation, Systems and Control Letters, 14, 55-61, 1990.

[PP2] E. Pardoux and S. Peng, Backward Doubly Stochastic Differential Equation and Quasi-linear PDEs, Lecture Notes in CIS, Vol. 176, Springger-verlag, 200-217, 1992.

[P1] S.Peng, BSDE and related $g$-expectation, Pitman Research Notes in Mathematics Series, No. 364, PP 141-159, 1997.

[P2] S. Peng, Monotonic limit theorem of BSDE and nonlinear decomposition theorem of Doob-Meyer type. Probab. Theory \& Rel. Fields, 113, 473-499, 1999.

[P3] S.Peng, Probabilistic interpretation for systems of quasilinear Parabolic probolic PDE and application, Stoch. and Stoch. Reports, 37, 61-74, 1991.

[MPY] J.Ma,J.Protter, J.Yong, Solving Forward-backward stochastic differential equationsa four step scheme, Proba. Theory and Related fields, 98, 339-359, 1994.

[S1] D. Schmeidler, Integral representation without additivity, Proceeddings of American Math. Society, Vol. 97, No. 2, p. 255-261, 1986.

[S2] D. Schmeidler, Subjetive probability and expected utility without additivity. Econometrica, 57, 571-587, 1989. 


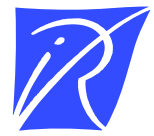

Unité de recherche INRIA Rocquencourt Domaine de Voluceau - Rocquencourt - BP 105 - 78153 Le Chesnay Cedex (France)

Unité de recherche INRIA Lorraine : LORIA, Technopôle de Nancy-Brabois - Campus scientifique 615, rue du Jardin Botanique - BP 101 - 54602 Villers-lès-Nancy Cedex (France)

Unité de recherche INRIA Rennes : IRISA, Campus universitaire de Beaulieu - 35042 Rennes Cedex (France)

Unité de recherche INRIA Rhône-Alpes : 655, avenue de l'Europe - 38330 Montbonnot-St-Martin (France)

Unité de recherche INRIA Sophia Antipolis : 2004, route des Lucioles - BP 93 - 06902 Sophia Antipolis Cedex (France)

INRIA - Domaine de Voluceau - Rocquencourt, BP 105 - 78153 Le Chesnay Cedex (France)

http://www.inria.fr

ISSN 0249-6399 